\title{
BETWEEN-FORMAT DIFFERENCES AND VARIABILITY OF TECHNICAL ACTIONS DURING SMALL-SIDED SOCCER GAMES PLAYED BY YOUNG PLAYERS
}

original paper

(1) University School of Physical Education in Wroclaw

DOI: https://doi.org/10.5114/hm.2018.83103

\author{
FILIPE MANUEL CLEMENTE ${ }^{1,2}$, YUNG-SHENG CHEN ${ }^{3}$, JOSE PEDRO BEZERRA ${ }^{1,4}$, \\ JOAO GUIOMAR ${ }^{1}$, RICARDO LIMA ${ }^{1}$ \\ ${ }^{1}$ Polytechnic Institute of Viana do Castelo, School of Sport and Leisure, Viana do Castelo, Portugal \\ ${ }^{2}$ Instituto de Telecomunicações, Covilhã, Portugal \\ ${ }^{3}$ Department of Exercise and Health Sciences, University of Taipei, Taipei, Taiwan \\ ${ }^{4}$ Research Center in Sports Sciences, Health Sciences and Human Development (CIDESD), UTAD University, Vila Real, \\ Portugal
}

\begin{abstract}
Purpose. This study purposes were: (i) to analyse variations of individual frequencies of technical actions between two small-sided game $(\mathrm{SSG})$ formats $(3 \times 3$ and $6 \times 6)$ in under- 8 soccer players; (ii) to test the variability of technical actions executed by very young players during $3 \times 3$ and $6 \times 6$ SSG formats.

Methods. Overall, 12 young male football players (age: $7.58 \pm 0.5$ years; practice: $2.5 \pm 0.5$ years) from the same Portuguese team voluntarily participated in the study. Both $3 \times 3$ and $6 \times 6$ formats were played twice, interspaced by one week, to test the variability of technical actions. Codification of actions involved conquered balls (CB), received balls (RB), lost balls (LB), attacking balls (AB), neutral balls (NB), not-succeeded shots (NSS), and succeeded shots (SS).

Results. The magnitudes of technical actions variability were small, with the exception of shots, which were moderately-tolargely variable. Between-formats analyses revealed almost certain very large decreases from $6 \times 6$ to $3 \times 3$ in the individual frequencies of RB (-45.7\% [-51.0; -39.9], ES (effect size): -4.69 [-5.48; -3.90]); CB (-42.3\% [-46.9; -37.3], ES: -2.32 [-2.68; $-1.97])$; LB (-59.9\% [-64.1; -55.2], ES: -3.69 [-4.13; -3.24]); NB (-37.6\% [-45.9; -27.9], ES: -1.76 [-2.29; -1.22]), and NSS $(-71.8 \%[-76.7 ;-65.9], E S:-5.74[-6.61 ;-4.88])$.
\end{abstract}

Conclusions. Passes, RB, and CB are reproducible but shots are variable across the sessions. Comparisons between formats clearly revealed that individual frequencies of technical actions largely increased in the $3 \times 3$ format.

Key words: association football, drill-based exercises, young, technical actions, SSG, variability

\section{Introduction}

Small-sided games (SSGs) are very popular drills used to promote the specificity of team sports exercises during training sessions $[1,2]$. The main purpose of these games is to simplify the complex formal game while maintaining the main properties and dynamics of the sport [3]. In soccer, these games are widely used to ensure the physical, physiological, and technical/ tactical stimulation of players [4]. The number of players, field dimensions, and modified rules are the main characteristics of SSGs that can be manipulated to fit the main objectives of the coach [5, 6].

The acute effects of SSGs in physiological responses and physical demands have been well-described in the recent decade, mainly in specialized youth and professional players [7-10]. The main evidence from previous studies suggests that smaller formats increase physiological intensity (e.g., heart rate, perceived exertion, and blood lactate concentration) and involve high numbers of accelerations and decelerations [11, 12]. Moreover, smaller formats also contribute to an

Correspondence address: Filipe Manuel Clemente, Complexo Desportivo e Lazer de Melgaço - Monte de Prado, 4960-320, Melgaço, Portugal, e-mail: filipe.clemente5@gmail.com

Received: December 3, 2018

Accepted for publication: January 29, 2019

Citation: Clemente FM, Chen Y-S, Bezerra JP, Guiomar J, Lima R. Between-format differences and variability of technical actions during small-sided soccer games played by young players. Hum Mov. 2018;19(5)special/issue:114-120 doi: https:// doi.org/10.5114/hm.2018.83103. 
increase in the individual frequencies of technical actions (e.g., passing, shots, received balls, dribbles) [13, 14]. Despite this evidence, there are very few studies dedicated to young novice players [15-17]. In the case of one study that reported the physical demands of a 4 vs. 4 format in under- 10 players, it was possible to observe values of distance covered between 294 and 380 meters, high speed running distance between 7 and 56 meters, and player load between 49 and 69 arbitrary units [17]. It was also verified that greater time-motion values obtained during games were positively and meaningfully associated with higher technical performance [17]. Also, a study revealed that very young players (under-11) executed more dribbles and delays in the $3 \times 3$ format in comparison with the $5 \times 5$ format, but more frequencies of unity and balance principles were observed in the bigger format [15]. In the same age category, it was verified that players tended to execute more aggressive tactical behaviours (as dribbles) during the $3 \times 3$ format and more safe behaviours during the $6 \times 6$ format [16]. This comparison of technical and tactical actions between formats should be increased, mainly because small and moderate game formats are widely used by coaches during training sessions.

Despite the pertinence of SSGs at both the novice and expert levels, there are some concerns regarding the variability of these games in their ability to properly stimulate the players $[18,19]$. Variability, in this context, refers to how spread out a set of technical actions or physiological measures is, and helps to describe how much data sets vary. Usually, this variability, in linear statistics (not considering the temporal series), can be calculated as a standardized measure of probability distribution or frequency distribution (coefficient of variation). Following that idea, some studies have aimed to test physiological and physical variability during SSGs [20, 21]. Generally, heart rate responses and low running intensities tend to reveal good levels of variability; however, blood lactate concentrations and high running speeds are too variable across games played in the same conditions for any meaningful conclusions to be drawn [18, 19]. A low variability level of SSGs is very important to ensure similar conditions of stimulation, especially in young players. However, to the best of our knowledge, only one study regarding reliability of performance in young players during SSGs has been conducted [22]. In that study, it was found that although some physical and physiological variables presented moderate to excellent reliability levels, tactical demands were not reliable [22]. The scarce number of studies conducted in the topic of SSGs, technical performance, and the young was recently highlighted in a systematic review about SSGs
[10]; it is important to increase and improve the understanding of those factors of the players' responses.

The identification of variations in technical actions between formats of play in young players is very important for coaches. Such evidence may help coaches in choosing the most appropriate format to fit the needs of players and may also contribute to the optimization of training plans and the reorganization of the design of SSGs to fit the technical aims of the coach. Moreover, an analysis of SSGs variability in very young players may add to our understanding of whether these games are useful and can be applied to ensure similar conditions of technical stimulation over time. A high level of technical variability may constrain the evolution of players during these games. For these reasons, the aim of this study was twofold: (i) to analyse the variations of individual frequencies of technical actions between two SSG formats $(3 \times 3$ and $6 \times 6)$ in under- 8 soccer players; and (ii) to test the variability of technical actions executed by very young players during $3 \times 3$ and $6 \times 6$ formats.

\section{Material and methods}

\section{Participants}

A total of 12 young male football players (age: $7.58 \pm$ 0.5 years; training experience: $2.5 \pm 0.5$ years) from the same Portuguese team voluntarily participated in the study. There were 4 defenders, 4 midfielders, and 4 forwards, equally distributed in 2 teams. The players trained twice a week (ca. 80 minutes per training session) and had one official match at the weekend. The youth team belongs to an academy of one team competing in the first league of Portugal. The subjects and their parents were informed about the study design and of the risks and benefits of participation.

\section{Experimental approach}

A repeated measures study was conducted during 4 consecutive weeks, aiming to analyse variations of technical actions between SSG formats and to test the variability of these games in young soccer players. The $3 \times 3$ and $6 \times 6$ formats (with small centred goals) were repeated with 1-week intervals to test the variability of technical actions in those games. The first 2 sessions ( 1 per week) were dedicated to the $3 \times 3$ format, and the $6 \times 6$ format was implemented during the following 2 weeks. The sessions were conducted in the second training session of each week (4 days after the last match and 2 days after the last training session). The sessions occurred at 6:30 p.m. at an av- 
F.M. Clemente, Y.-S. Chen, J.P. Bezerra, J. Guiomar, R. Lima, Variability of SSGs in young players

erage temperature of $13^{\circ} \mathrm{C}$, with no rain. The same players acted in the same balanced teams (based on the recommendations of the head coach and on playing positions), in the conditions similar between repeated measures. The games were recorded by 2 digital cameras, and technical actions were compared between sessions.

\section{Small-sided games}

Both formats $(3 \times 3$ and $6 \times 6)$ were played on a synthetic turf with the following dimensions: (i) $15 \times 20 \mathrm{~m}$ for the $3 \times 3$ format (ca. $50 \mathrm{~m}^{2}$ per player), and (ii) $22 \times 30 \mathrm{~m}$ for the $6 \times 6$ format (ca. $55 \mathrm{~m}^{2}$ per player). The formats were played with a small goal $(2 \times 1 \mathrm{~m})$, positioned in the centre of each ending line. Both formats were played with a training regimen of 3 sets of 3 minutes with a resting period of 2 minutes between sets. Two assistant coaches were placed around the pitch to quickly replace the balls that crossed the sidelines. The players were previously familiarized with the training formats to ensure the knowledge of dynamics involved in these games. Prior to all SSGs, a standardized warm-up protocol was implemented consisting of 5 minutes of jogging, 5 minutes of dynamic stretching and mobility, and 5 minutes of sprints, accelerations, and decelerations. No information or feedback about strategy or tactics were provided to players.

\section{Technical actions}

Two digital cameras (GoPro Hero 2, $1280 \times 960$, $25 \mathrm{~Hz}$ ) - one positioned above the pitch in an open angle and the other focusing on the player in possession of the ball - recorded the matches. The observation of the technical actions was processed on the basis of the following codification: conquered balls (CB), consisting of recovered balls that a player made against an opponent; received balls (RB), consisting of actions when a player received a pass from a teammate; lost balls (LB), recorded when a player lost control of the ball to an opponent or by going out of bounds; attacking balls (AB), consisting of passes that moved the ball forward; neutral balls (NB), recorded when a player made a pass that did not move the ball forward; notsucceeded shots (NSS), consisting of shots that were not goals; and succeeded shots (SS), consisting of shots that resulted in a goal scored against the opponent. The codification followed the performance categories presented by Gréhaigne et al. [23].

Notification and codification were performed by the same observer, who had more than 3 years of experience in soccer analysis. The observer was previ- ously tested (observing and coding 3 matches supervised for senior researchers in observational analysis) for the capacity to properly code the variables, namely by comparing the results with other observers and revealing a high-level of reliability (intra-class correlation coefficient: > 0.90) and accuracy (96\% of the right coding, considering the codification of the senior observers). A test-retest interspaced by 20 days with $10 \%$ of the data was executed to analyse the reliability of the observer. The intra-class correlation test revealed a mean level of 0.91 (excellent reliability), thus ensuring enough reliability for the researchers to move forward with the analysis. The intra-class correlation test reported the following values per each measurement analysed: RB: 0.95; CB: 0.90; LB: 0.89; AB: 0.91; NB: 0.89; NSS: 0.90; and SS: 0.93 .

\section{Statistical procedures}

The results were presented in the form of text, a table, and a figure, as either means with a standard deviation $(S D)$ or means with a $90 \%$ confidence interval $(90 \% \mathrm{CI})$ where specified. Between-session variability of technical actions in each set and their accumulated values derived from all sets were analysed by computing typical errors of measurement, expressed as a coefficient of variation $(C V)$ or as standardized typical error (STE), with the use of a specifically-designed spreadsheet [24]. To investigate the within-session variations, differences between formats were analysed with standardized differences of effect size (ES) with a 90\% CI [25]. The following interpretation of ES was used [26]: < 0.2 : trivial; 0.2-0.59: small; 0.6-1.19: moderate; $\geq 1.2$ : large. Probabilities were calculated with the consideration of the smallest worthwhile changes (SWC, $0.2 \times$ between-subject SD) [27]. Qualitative probabilistic mechanistic inferences about the true effects were based on these probabilities [27]. The scale for qualitative probabilities was as follows: > 25-75\%: possible; > 75-95\%: likely; > 95-99\%: very likely; > 99\%: almost certain [27].

\section{Ethical approval}

The research related to human use has been complied with all the relevant national regulations and institutional policies, has followed the tenets of the Declaration of Helsinki, and has been approved by the authors' institutional review board or an equivalent committee.

\section{Informed consent}

Informed consent has been obtained from the legal guardians of all individuals included in this study. 


\section{Results}

The variability analysis of the sum of technical actions per session can be found in Table 1. Moderate variability in NSS (CV, 19.8; 90\% CI [14.5; 32.3]; STE, $0.75[0.56 ; 1.16])$ and large variability in SS (CV, 51.9 [36.7; 91.2]; STE, 1.22 [0.92; 1.90]) were observed during $3 \times 3$ format. In the case of $6 \times 6$ format, moderate variabilities were noted in $\mathrm{RB}(\mathrm{CV}, 12.1$ [8.9; 19.4]; STE, 0.67 [0.50; 1.04]), NSS (CV, 41.5 [29.7; 71.4]; STE, 0.78 [0.59; 1.22]), and SS (CV, 43.6 [31.1; 75.3]; STE, 1.15 [0.86; 1.79]). The remaining variabilities in $3 \times 3$ and $6 \times 6$ formats were small.

Between-format differences considering the average of the sums of the technical actions per session are presented in Figure 1. Almost certain very large decreases of $6 \times 6$ vs. $3 \times 3$ were observed in the individual frequencies of RB (-45.7\% [-51.0; -39.9], ES: -4.69 [-5.48; $-3.90]) ;$ CB $(-42.3 \%$ [-46.9; -37.3$]$, ES: -2.32 [-2.68; $-1.97]) ; \mathrm{LB}(-59.9 \%$ [-64.1; -55.2$]$, ES: -3.69 [-4.13; $-3.24]) ; \mathrm{NB}(-37.6 \%$ [-45.9; -27.9$], E S:-1.76$ [-2.29; $-1.22])$, and NSS (-71.8\% [-76.7; -65.9], ES: -5.74 [-6.61; $-4.88])$. Very likely moderate decreases of $6 \times 6$ vs. $3 \times 3$ were found in the individual frequencies of $\mathrm{AB}$ $(-23.4 \%[-32.8 ;-12.5], E S:-1.16[-1.74 ;-0.58])$. Finally, likely small decreases of $6 \times 6$ vs. $3 \times 3$ were observed in the individual frequencies of SS $(-24.0 \%[-38.4$; -6.3], ES: -0.51 [-0.91; -0.12]).

Table 1 . Variability of the sum of technical actions ( 3 sets) between sessions during $3 \times 3$ and $6 \times 6$ formats

\begin{tabular}{|c|c|c|c|c|c|c|c|c|}
\hline \multirow{2}{*}{ Format } & \multirow{2}{*}{ Variable } & \multirow{2}{*}{$\begin{array}{c}M(S D) \\
\text { Sum for } \\
\text { session } 1\end{array}$} & \multirow{2}{*}{$\begin{array}{c}M(S D) \\
\text { Sum for } \\
\text { session } 2\end{array}$} & \multicolumn{2}{|c|}{ Typical error $(C V)$} & \multicolumn{2}{|c|}{ Standardized typical error } & \multirow{2}{*}{ Magnitude } \\
\hline & & & & Value & $90 \% \mathrm{CI}$ & Value & $90 \% \mathrm{CI}$ & \\
\hline \multirow{7}{*}{$3 \times 3$} & $\mathrm{RB}$ & $30.0(4.3)$ & $30.3(3.5)$ & 6.4 & {$[4.7 ; 10.1]$} & 0.48 & {$[0.36 ; 0.74]$} & Small \\
\hline & $\mathrm{CB}$ & $11.1(2.4)$ & $11.0(2.7)$ & 9.7 & {$[7.2 ; 15.4]$} & 0.40 & {$[0.30 ; 0.62]$} & Small \\
\hline & $\mathrm{LB}$ & $12.2(2.8)$ & $10.8(2.1)$ & 11.5 & {$[8.5 ; 18.4]$} & 0.45 & {$[0.34 ; 0.70]$} & Small \\
\hline & $\mathrm{AB}$ & $11.9(2.8)$ & $13.2(2.6)$ & 12.3 & {$[9.0 ; 19.7]$} & 0.50 & {$[0.37 ; 0.77]$} & Small \\
\hline & NB & $8.7(2.2)$ & $8.8(2.6)$ & 13.1 & {$[9.6 ; 21.0]$} & 0.47 & {$[0.35 ; 0.72]$} & Small \\
\hline & NSS & $7.0(1.8)$ & $7.8(1.5)$ & 19.8 & {$[14.5 ; 32.3]$} & 0.75 & {$[0.56 ; 1.16]$} & Moderate \\
\hline & SS & $1.3(0.5)$ & $1.3(0.5)$ & 51.9 & {$[36.7 ; 91.2]$} & 1.22 & {$[0.92 ; 1.90]$} & Large \\
\hline \multirow{7}{*}{$6 \times 6$} & $\mathrm{RB}$ & $15.3(3.0)$ & $17.6(2.2)$ & 12.1 & {$[8.9 ; 19.4]$} & 0.67 & {$[0.50 ; 1.04]$} & Moderate \\
\hline & $\mathrm{CB}$ & $6.9(1.4)$ & $5.9(2.0)$ & 18.2 & {$[13.3 ; 29.6]$} & 0.58 & {$[0.43 ; 0.90]$} & Small \\
\hline & $\mathrm{LB}$ & $4.7(1.1)$ & $4.6(1.2)$ & 12.1 & {$[8.9 ; 19.4]$} & 0.42 & {$[0.31 ; 0.64]$} & Small \\
\hline & $\mathrm{AB}$ & $9.4(3.1)$ & $10.1(2.4)$ & 11.1 & {$[8.2 ; 17.8]$} & 0.37 & {$[0.27 ; 0.57]$} & Small \\
\hline & NB & $5.3(1.7)$ & $5.8(1.7)$ & 9.2 & {$[6.8 ; 14.6]$} & 0.27 & {$[0.20 ; 0.42]$} & Small \\
\hline & NSS & $2.3(0.9)$ & $2.4(1.0)$ & 41.5 & {$[29.7 ; 71.4]$} & 0.78 & {$[0.59 ; 1.22]$} & Moderate \\
\hline & SS & $1.3(0.5)$ & $1.3(0.5)$ & 43.6 & {$[31.1 ; 75.3]$} & 1.15 & {$[0.86 ; 1.79]$} & Moderate \\
\hline
\end{tabular}

$\mathrm{RB}$ - received balls, CB - conquered balls, LB - lost balls, AB - attacking balls, NB - neutral balls, NSS - not-succeeded shots, SS - succeeded shots, $M$ - mean, $S D$ - standard deviation, $C V$ - coefficient of variation (\%), 90\% CI - confidence interval of $90 \%$

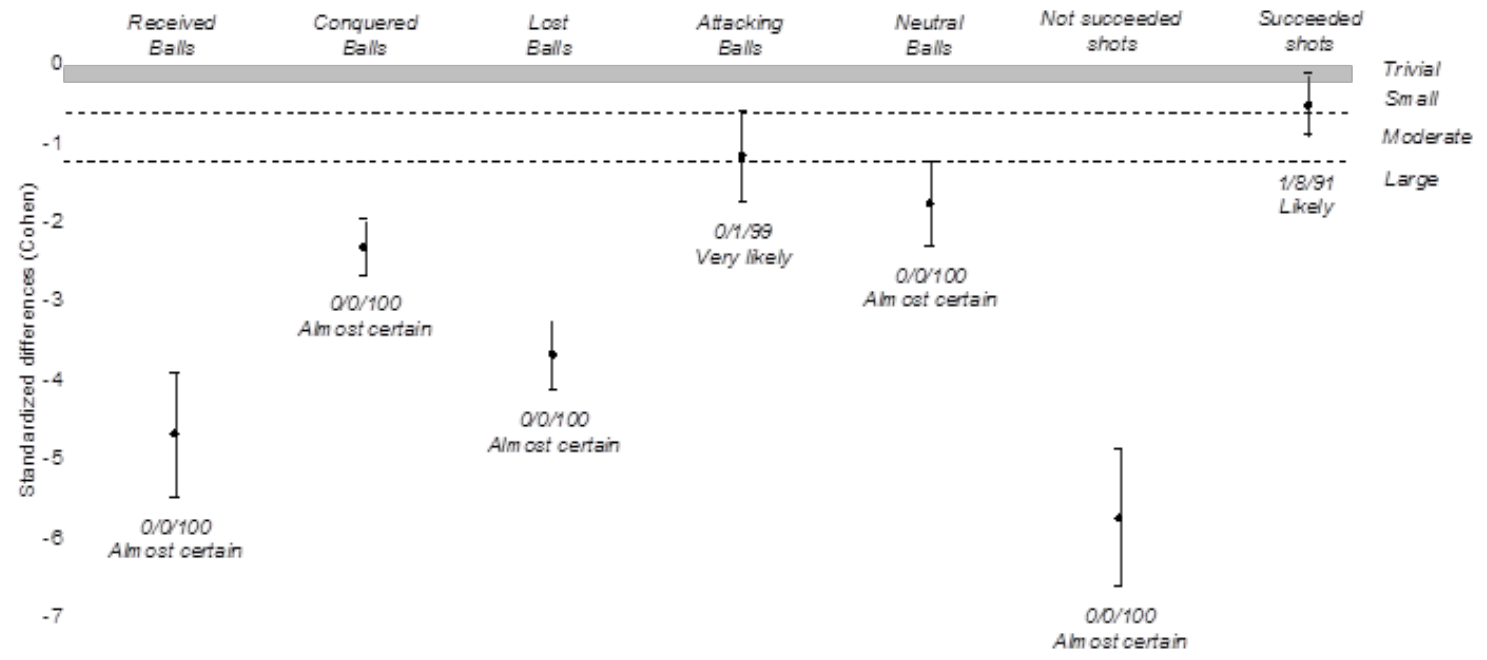

Figure 1. Standardized difference (Cohen) between formats in received balls, conquered balls, lost balls, attacking balls, neutral balls, not-succeeded shots, and succeeded shots. Grey area represents the trivial magnitude. Standardized value direction depends on the relationship between $6 \times 6$ and $3 \times 3$ 
F.M. Clemente, Y.-S. Chen, J.P. Bezerra, J. Guiomar, R. Lima, Variability of SSGs in young players

\section{Discussion}

The study tested the variability of technical actions during SSGs played by young players. The STE revealed small magnitudes of variability for all technical actions, with the exception of shots. NSS and SS were moderately-to-largely variable in both $3 \times 3$ and $6 \times 6$ formats. Considering the reality of the match, it would be possible to hypothesize a greater variability of shots as such events are highly dependent on the prominence of the players in the match and the dynamics emerged from the cooperation and opposition relationships [28]. In a study conducted among youth soccer players aiming to analyse the capacity of SSGs to assess skill proficiency, it was found that $S D$ s represented almost half of the average of attempted shots and almost the same value for the completed shots, being much greater than the $S D$ s of the other technical actions reported [29]. In our study, the $C V$ tested as typical error revealed values of NSS of $19.8 \%$ and $41.5 \%$ and values of SS of $51.9 \%$ and $43.6 \%$ for the $3 \times 3$ and $6 \times 6$ formats, respectively, considering that the same players were tested in both formats. Such values suggest that shots are not reproducible during SSGs, and for that reason, coaches should be aware that this fact could be important to create complementary task constraints to ensure better levels of variability or to opt for analytical exercises to ensure similar conditions of exercising of this technical action [5].

In the case of the remaining technical actions (RB, $\mathrm{CB}, \mathrm{LB}, \mathrm{AB}$, and $\mathrm{NB}$ ), small values of STE were verified, thus ensuring interesting levels of variability during the $3 \times 3$ and $6 \times 6$ formats. Previous studies that used SSGs to assess skill proficiency in youth players reported that passes and touches were the actions with small $S D$ s in comparison with the averages [29], thus confirming that SSGs can be implemented for the objective of reproducing passes and RB. Results regarding variability provided by the present study suggest that coaches can use SSGs to ensure stable levels of passes, $\mathrm{RB}$, or $\mathrm{CB}$, ensuring that players will be stimulated in the same way across sessions.

The second purpose of our study was to compare the individual frequencies of technical actions executed by very young players during $3 \times 3$ and $6 \times 6$ formats. Results revealed almost certain large increases of individual frequencies in RB (45.7\%), CB (42.3\%), LB (59.9\%), NB (37.6\%), and NSS (71.8\%) during the smaller format $(3 \times 3)$. Moreover, very likely moderate increases in $\mathrm{AB}(23.4 \%)$ and likely small increases in SS (24\%) were found in the $3 \times 3$ format. These inferences are in line with the findings of previous re- search that tested technical actions in different SSGs formats, revealing that smaller formats tended to increase individual participation [12, 30, 31]. AB (passes moving forward) and SS were the technical actions with lower differences in terms of magnitude, which probably suggests that these critical attacking processes are more stable, independent of the format of play. Such evidence may reveal that coaches can organize medium formats $(6 \times 6)$ to create similar conditions of critical attacking actions, such as passing, moving forward, and SS. However, if a coach wants to develop other actions, such as passes backwards or sideways or CB, smaller formats should be preferred.

This study had some limitations. The small sample should be considered among the main ones. However, the magnitudes observed provide some confidence in terms of the main evidence for coaches. Moreover, tactical behaviours were not analysed. In this regard, it would be interesting to associate the technical actions with the behaviours of very young players. For that reason, future studies should analyse specific tactical behaviours and measure their variability, as well as investigate their variations between formats. Finally, there were 2 weeks of difference between formats, possibly affecting the inter-format analysis. In the future, a mixed design should be considered to avoid the possible learning effect.

The main evidence of this study may provide practical implications for coaches. The study revealed that $3 \times 3$ and $6 \times 6$ SSGs generated reproducible individual frequencies of technical actions, with the exception of shots in novice and young soccer players. For that reason, coaches may use SSGs to stabilize technical stimuli across sessions. However, more task constraints or new drills should be organized to ensure the proper stimulation of shots. Beyond these applications, the present study also revealed that the $3 \times 3$ format moderately-to-largely increased the individual frequencies of defensive and attacking technical actions. For that reason, children soccer coaches should use the $3 \times 3$ format when the intention is merely to promote individual technical actions in offensive and defensive phases. It is important to highlight that the results obtained from the study should not discourage the use of SSGs in children. However, SSGs should be understood as a component of training associated with a specialization (e.g., deliberate practice), contributing to a balanced intervention in the development of talented soccer players [32], which must also contain a diversification component and some specific and close skill-based tasks to balance the variability effect of SSGs in some technical aspects. 


\section{Conclusions}

The results of this study revealed that $\mathrm{RB}, \mathrm{CB}, \mathrm{LB}$, and passes (moving forward, backward, or sideward) presented a low variability level during $3 \times 3$ and $6 \times 6$ SSGs played by young soccer players. However, high levels of variability were observed in shots; thus, it would possibly be advisable not to stimulate the same players equally during SSGs. The second purpose of the study also confirmed previous results in revealing that the $3 \times 3$ format clearly moderately-tolargely increased individual frequencies of both defensive and technical actions in comparison with the $6 \times 6$ format.

\section{Disclosure statement}

No author has any financial interest or received any financial benefit from this research.

\section{Conflict of interest}

The authors state no conflict of interest.

\section{References}

1. Halouani J, Chtourou H, Gabbett T, Chaouachi A, Chamari K. Small-sided games in team sports training: a brief review. J Strength Cond Res. 2014;28(12):35943618; doi: 10.1519/JSC.0000000000000564.

2. Clemente FM, Martins FM, Mendes RS. Developing aerobic and anaerobic fitness using small-sided soccer games: methodological proposals. Strength Cond J. 2014; 36(3):76-87; doi: 10.1519/SSC.0000000000000063.

3. Davids K, Araújo D, Correia V, Vilar L. How small-sided and conditioned games enhance acquisition of movement and decision-making skills. Exerc Sport Sci Rev. 2013;41(3):154-161; doi: 10.1097/JES.0b013e31829 $2 \mathrm{f} 3 \mathrm{ec}$.

4. Hill-Haas SV, Dawson B, Impellizzeri FM, Coutts AJ. Physiology of small-sided games training in football: a systematic review. Sports Med. 2011;41(3):199-220; doi: 10.2165/11539740-000000000-00000.

5. Clemente FM. Small-sided and conditioned games in soccer training: the science and practical applications. Singapore: Springer; 2016.

6. Clemente FM, Nikolaidis PT, van der Linden CMIN, Silva B. Effects of small-sided soccer games on internal and external load and lower limb power: a pilot study in collegiate players. Hum Mov. 2017;18(1):50-57; doi: 10.1515/humo-2017-0007.

7. Köklü Y, Aşçi A, Koçak FÜ, Alemdaroğlu U, Dündar U. Comparison of the physiological responses to different small-sided games in elite young soccer players. J Strength Cond Res. 2011;25(6):1522-1528; doi: 10.1519/ JSC.0b013e3181e06ee1.

8. Owen AL, Wong del P, McKenna M, Dellal A. Heart rate responses and technical comparison between small- vs. large-sided games in elite professional soccer. J Strength Cond Res. 2011;25(8):2104-2110; doi: 10.1519/JSC.0b013e3181f0a8a3.

9. Lacome M, Simpson BM, Cholley Y, Lambert P, Buchheit M. Small-sided games in elite soccer: does one size fit all? Int J Sports Physiol Perform. 2018;13(5):568576; doi: 10.1123/ijspp.2017-0214.

10. Sarmento H, Clemente FM, Harper LD, da Costa IT, Owen A, Figueiredo AJ. Small sided games in soccer - a systematic review. Int J Perform Anal Sport. 2018; 18(5):693-749; doi:10.1080/24748668.2018.1517288.

11. Williams K, Owen A. The impact of player numbers on the physiological responses to small sided games. J Sports Sci Med. 2007;6(10):99-102.

12. Clemente FM, Wong del P, Martins FM, Mendes RS. Acute effects of the number of players and scoring method on physiological, physical, and technical performance in small-sided soccer games. Res Sports Med.2014;22(4):380-397; doi: 10.1080/15438627.2014. 951761.

13. Dellal A, Owen A, Wong DP, Krustrup P, van Exsel M, Mallo J. Technical and physical demands of small vs. large sided games in relation to playing position in elite soccer. Hum Mov Sci. 2012;31(4):957-969; doi: 10.1016/ j.humov.2011.08.013.

14. Owen AL, Wong DP, Paul D, Dellal A. Physical and technical comparisons between various-sided games within professional soccer. Int J Sports Med. 2014; 35(4):286-292; doi: 10.1055/s-0033-1351333.

15. Castelão D, Garganta J, Santos R, Teoldo I. Comparison of tactical behaviour and performance of youth soccer players in 3v3 and 5v5 small-sided games. Int J Perform Anal Sport. 2014;14(3):801-813; doi: 10.1080/ 24748668.2014.11868759.

16. Silva B, Garganta J, Santos R, Teoldo I. Comparing tactical behaviour of soccer players in 3 vs. 3 and 6 vs. 6 small-sided games. J Hum Kinet. 2014;41(1):191-202; doi: 10.2478/hukin-2014-0047.

17. Fenner JS, Iga J, Unnithan V. The evaluation of smallsided games as a talent identification tool in highly trained prepubertal soccer players. J Sports Sci. 2016; 34(20):1983-1990; doi: 10.1080/02640414.2016.1149602.

18. Hill-Haas S, Coutts A, Rowsell G, Dawson B. Variability of acute physiological responses and performance profiles of youth soccer players in small-sided games. J Sci Med Sport. 2008;11(5):487-490; doi: 10.1016/j. jsams.2007.07.006.

19. Stevens TG, De Ruiter CJ, Beek PJ, Savelsbergh GJ. Validity and reliability of 6-a-side small-sided game locomotor performance in assessing physical fitness in football players. J Sports Sci. 2016;34(6):527-534; doi: 10.1080/02640414.2015.1116709.

20. Rampinini E, Impellizzeri FM, Castagna C, Abt G, Chamari K, Sassi A, et al. Factors influencing physiological responses to small-sided soccer games. J Sports Sci. 2007;25(6):659-666; doi: 10.1080/02640410600 811858 . 


\section{HUMAN MOVEMENT}

F.M. Clemente, Y.-S. Chen, J.P. Bezerra, J. Guiomar, R. Lima, Variability of SSGs in young players

21. Ngo JK, Tsui MC, Smith AW, Carling C, Chan GS, Wong del P. The effects of man-marking on work intensity in small-sided soccer games. J Sports Sci Med. 2012;11(1):109-114.

22. Bredt SGT, Praça GM, Figueiredo LS, de Paula LV, Silva PCR, de Andrade AGP, et al. Reliability of physical, physiological and tactical measures in small-sided soccer games with numerical equality and numerical superiority. Rev Bras Cineantropom Desempenho Hum. 2016;18(5):602-610; doi: 10.5007/1980-0037. 2016v18n5p602.

23. Gréhaigne JF, Godbout P, Bouthier D. Performance assessment in team sports. J Teach Phys Educ. 1997; 16(4):500-516; doi: 10.1123/jtpe.16.4.500.

24. Hopkins WG. Spreadsheets for analysis of validity and reliability. Sportscience. 2015;19:36-44.

25. Cohen J. Statistical power analysis for the behavioral sciences. New York: Lawrence Erlbaum Associates; 1988.

26. Batterham AM, Hopkins WG. Making meaningful inferences about magnitudes. Int J Sports Physiol Perform. 2006;1(1):50-57; doi: 10.1123/ijspp.1.1.50.

27. Hopkins WG, Marshall SW, Batterham AM, Hanin J. Progressive statistics for studies in sports medicine and exercise science. Med Sci Sports Exerc. 2009;41(1): 3-13; doi: 10.1249/MSS.0b013e31818cb278.

28. Sarmento H, Clemente FM, Araújo D, Davids K, McRobert A, Figueiredo A. What performance analysts need to know about research trends in association football (2012-2016): a systematic review. Sports Med. 2018; 48(4):799-836; doi: 10.1007/s40279-017-0836-6.

29. Bennett KJM, Novak AR, Pluss MA, Stevens CJ, Coutts AJ, Fransen J. The use of small-sided games to assess skill proficiency in youth soccer players: a talentidentification tool. SciMed Football. 2018;2(3):231236; doi: 10.1080/24733938.2017.1413246.

30. Almeida CH, Ferreira AP, Volossovitch A. Offensive sequences in youth soccer: effects of experience and small-sided games. J Hum Kinet. 2013;36(1):97-106; doi: 10.2478/hukin-2013-0010.

31. Joo CH, Hwang-Bo K, Jee H. Technical and physical activities of small-sided games in young Korean soccer players. J Strength Cond Res. 2016;30(8):21642173; doi: 10.1519/JSC.0000000000001319.

32. Sarmento H, Anguera MT, Pereira A, Araújo D. Talent identification and development in male football: a systematic review. Sports Med. 2018;48(4):907-931; doi: 10.1007/s40279-017-0851-7. 